\title{
ECONOMIA DAS PEQUENAS EMPRESAS: ASPECTOS ECONÔMICOS E DE GESTÃO
}

\author{
Valdemiro Hildebrando ${ }^{1}$
}

\section{INTRODUÇÃO}

Discutir a relevância econômica das pequenas e médias empresas (PMEs) é ajudar a reformar o paradigma que beneficia a grande empresa que, por meio da economia de escala, tem sido mostrada - teoricamente falando como de relevância superior em termos de geração de renda e emprego. Dentre as causas principais do altíssimo nível de mortalidade entre as PMEs, fatores financeiros e de gestão são abordados. Tais assuntos são, em resumo, o objetivo deste estudo.

Nas duas últimas décadas, a importância das PMEs passou a ser considerada como um assunto sério por universidades, pesquisadores e políticos, a julgar pelo número de cursos criados, novas revistas científicas e artigos publicados a respeito, e também pela importância dada ao empreendedorismo (BROCK e EVANS, 1989; ACS, 1992; JULIEN, 1993; 1998; LIEDHOLM e MEAD, 1999; TOMMASO e DUBBINI, 2000, e vários outros). Alguns acadêmicos vêem pequenos negócios e empreendedorismo incluídos entre as mais importantes disciplinas da área de negócios deste século (SOLOMON et al., 1994; GRANT, 1998).

Entretanto, alguns microeconomistas e outros profissionais da área ainda vêem pequenos negócios apenas como um grupo informal de empresas, um agregado econômico mencionado por Machlup (1967) que merece atenção apenas quando estas miríades de pequenas empresas tornam-se grandes (JULIEN, 1998). Nos países em desenvolvimento, a presença dos pequenos negócios foi interpretada como característica de subdesenvolvimento (TOMMASO et al., 2000). No passado recente, economistas

${ }^{1}$ Ph.D Universidade do Planalto Catarinense. e-mail: brandovb@yahoo.com - URL: http://uniplac.net/ vbrando 
insistiam que, de acordo com a teoria neoclássica das economias de escala, pequenas empresas eram menos eficientes que as maiores em muitos aspectos (KIRCHHOFF, 1991; ACS, 1992). A percepção de que pequenos negócios eram algo "not serious, slightly eccentric" (JULIEN, 1998, p. 2) continuou até meados dos anos 1970 e lentamente começou a mudar até o final do século, quando este campo de estudos passou por uma explosão, em sentido positivo. Os debates sobre o tamanho da empresa "triggered unexpected reappraisal of the role and importance of small manufacturing firms" (ACS, 1992, p. 38).

As causas para o renascimento das PMEs são identificáveis: depois do choque do petróleo de 1973, o mercado presenciou casos espetaculares de grandes empresas com graves problemas financeiros, fazendo o permanente problema do desemprego ainda pior. $\mathrm{O}$ governo e suas empresas, especialmente onde as estatais eram as indiscutíveis líderes do mercado, ${ }^{2}$ reagiram passivamente ao desastroso ambiente econômico, e os resultados desta reação não foram suficientes para ultrapassar este período sem provocar ainda maior turbulência e, com os aumentos dos custos, os pesados déficits operacionais tiveram de ser cobertos com transferências dos cofres públicos.

As PMEs, por sua vez, pareciam atravessar esses tempos difíceis sem maiores problemas, um fenômeno que ocorreu praticamente em todos os lugares no mundo desenvolvido e em desenvolvimento. Um artigo publicado por Birch (1979) afirmava que as PMEs criavam a maioria dos novos empregos nos Estados Unidos. Apesar de que os dados utilizados não eram inteiramente precisos e foram posteriormente refinados pelo autor e outros pesquisadores, o estudo provocou “...an enormous controversy... Birch's findings violated a widely-held set of prior beliefs" (PIORE, 1990, cap. 7). A pesquisa foi um passo fundamental para um novo entendimento do papel das PMEs na economia.

Finalmente, a Organização para o Desenvolvimento e Cooperação Econômica (OECD) publicou um estudo em 1985 concluindo que, em diversos países europeus, a tendência para a concentração dos trabalhadores em pequenas empresas podia ser verificada. Economistas, políticos e a imprensa abraçaram esta idéia (BROCK et al., 1989; JULIEN, 1998). Um novo

${ }^{2}$ No Brasil, desde as primeiras tentativas de acelerar o processo de industrialização nos anos 1950, e especialmente nos anos 1960, um período de profundas mudanças na economia brasileira, o governo colocou ênfase total no desenvolvimento de grupos econômicos nacionais e estatais, e as grandes empresas foram estimuladas a crescer para enfrentar a competição externa. Deste modo, não havia espaço para as PMEs. 
paradigma se impunha. Grandes empresas, estatais e multinacionais que representavam o carro-chefe da economia, estavam sendo substituídas pelas PMEs, sinônimos de flexibilidade e inovação (SCHERER, 1980; AUDRETSCH, 1995, entre outros).

Loveman et al. (1990, p. 4) advertem que "it is important to guard against rushing into premature and general conclusions... of the shift toward smaller units". Qualquer espécie de determinismo físico não pode explicar a superioridade de um determinado setor econômico; as grandes companhias tinham em seu favor a teoria da economia de escala, a forte posição em termos de participação no mercado, gastos em treinamento de pessoal, pesquisa e desenvolvimento de novos produtos, publicidade e mais eficientes canais de distribuição.

A geração de emprego, no nível das PMEs, foi inicialmente considerada uma simples transferência de recursos humanos das grandes empresas. Deste modo, no nível macro, estas mudanças estruturais não seriam tão importantes como pareciam. Segundo, os sindicatos não aceitaram tão rapidamente a idéia de que a estrutura do emprego tinha mudado das grandes empresas - onde a influência e o poder dos sindicatos eram importantes para as pequenas, uma situação que poderia levar a uma mudança negativa no equilíbrio das relações industriais (LOVEMAN et al., 1990). As complexas inter-relações estabelecidas pelas PMEs na sociedade podem explicar parte da sua pretensa superioridade em termos de flexibilidade e mudança, como também a organização social e política na comunidade (PIORE e SABEL, 1984).

Outros fatores devem ser também considerados. Primeiro, em muitos mercados, as políticas deregulatórias impostas pelos governos, uma decisão em parte alimentada pelas pessoas que se opunham à noção de intervenção governamental na economia (PIORE, 1990). Segundo, a remoção de barreiras de entrada (LOVEMAN et al., 1990) que impediam as novas empresas de entrar em mercados importantes como os de exportação, produtos microeletrônicos e de tecnologia de ponta, etc. Terceiro, o decisivo apoio financeiro do governo foi uma mudança importante, porque no passado o suporte financeiro era destinado quase exclusivamente às grandes companhias, multinacionais e estatais (SEBRAE, 1994). Por fim, Acs (1992) menciona a necessidade de especialização flexível para responder a necessidades especiais dos consumidores, e redes de fornecedores especializados, um aspecto mencionado posteriormente em Arzeni (1997). Carrée e Thurik (2002, p. 3), citando um trabalho anterior de Loveman et al. (1990), mencionam a recente reestruturação industrial com a formação de novas comunidades de negócios, centralização e desintegração vertical como causas para a ascensão das pequenas empresas. 
Deste modo, o novo paradigma mostrava que as PMEs eram melhores para os mercados competitivos, e o seu desempenho superior ao das grandes empresas em termos de geração de emprego, assim merecendo menos regulação e mais incentivos (LOVEMAN et al., 1990). Com algum humor, Scherer (1980), citado por Brock et al., 1989, p. 13, conclui que "a little bit of bigness is good for invention and innovation." Entretanto, somente um pouquinho, porque depois de um certo limite, "further bigness adds little or nothing".

Potobsky (1992), num extenso trabalho sobre PMEs e as leis trabalhistas, menciona que as microempresas nos países em desenvolvimento têm uma característica especial, a de compor o assim chamado setor informal, definido como as micro e pequenas unidades produtoras e distribuidoras de bens econômicos. Elas operam com pouco ou nenhum capital, usam baixo nível de tecnologia, e usualmente a sua importância decorre do fato de que controles burocráticos são abolidos porque caros e ineficientes. Entretanto, sua afirmativa de que o setor informal não é um fenômeno associado com estratégias de sobrevivência em países pobres ou em desenvolvimento é equivocada. Este tipo de economia subterrânea tem sido uma resposta para a falta crônica de investimentos e oportunidades e também para o (medo de) desemprego (ARRIGHETTI e VIVARELLI, 1999). Alguns economistas acreditam que o impacto da informalidade na economia brasileira é significante, em torno de um terço do universo tributável das empresas. A importância socioeconômica, como um redutor dos efeitos do desemprego, é ainda maior. Solimeo (1994, p. 9) argumenta os "custos de formalização" e a legislação salarial / trabalhista como obstáculos às PMEs no Brasil; Lessa (2004, p. A11) denuncia que as PMEs são "o domínio da informalidade", e esta uma resposta à tributação excessiva (ARZENI, 1997).

Audrestch (1990) identificou, entre outros fatores, os assim chamados "baby-boomers" e mulheres nos Estados Unidos como geradores de uma grande mudança no mercado de trabalho depois dos anos 50, uma idéia compartilhada por Dennis, Philips e Starr (1994). Como não havia empregos suficientes para todos, muitos tiveram de descobrir oportunidades de autoemprego, assim contribuindo para a explosão dos pequenos negócios. 


\section{RELEVÂNCIA ECONÔMICA DAS PMES}

Julien (1998, p. 7), cujo trabalho é utilizado extensamente aqui, argumenta que tipologias quantitativas para os pequenos negócios

resultam da abordagem econômica tradicional... são as mais facilmente utilizadas, tendem a serem usadas como critério de qualificação para programas de apoio governamental, e freqüentemente são os primeiros passos para identificar amostragens que serão subseqüentemente utilizadas em maiores detalhes. ${ }^{3}$

Outros países também adotaram diferentes critérios para definir tamanho, apesar da advertência de Loveman et al. (1990 p. 4): "the concern with the scale of enterprises... is meaningful only in relative or comparative context." Nos Estados Unidos, Piore (1990, p. 69) discute que o pequeno negócio é

salient in American political rhetoric, but... it is not a distinct category in the actual organization of the U.S., politically, economically, or socially, and it has achieved only limited and precarious recognition as a category of social and economic legislation.

Entretanto, os números são impressionantes. Nos Estados Unidos, mais de 23,2 milhões de retornos de imposto de renda foram protocolados em 1996 e deste montante mais de $99 \%$ referem-se a pequenos negócios; 64\% dos 2,5 milhões de novos empregos criados; $53 \%$ do emprego em geral, e em torno de $47 \%$ do PIB (U.S.SBA, 1997).

Sebrae (1994) relata que o pequeno negócio gerou 53\% do PIB brasileiro, e sua parte no emprego é igualmente impressionante (em torno de $59 \%$ da força de trabalho do país e $42 \%$ de todos os salários pagos), sem considerar o impacto do assim chamado setor informal numa economia profundamente atingida por altas taxas de desemprego. No interior rural brasileiro, nas pequenas comunidades largamente baseadas em atividades rurais e de agricultura, pequenos negócios são também dominantes. Nos centros urbanos, com os programas de estabilização nas duas últimas décadas objetivando o controle inflacionário e a redução de disparidades setoriais,

${ }^{3}$ Tradução do autor. 
os jovens têm de encontrar empregos fora das áreas tradicionais do governo e grandes negócios, assim tornando-se, ao final, auto-empregados e donos de pequenos negócios, e também empreendedores (SEBRAE, 1994).

Gorton (1999) menciona que, no começo dos anos 1990, mais de $50 \%$ da população na Bolívia, Colômbia, Equador e Peru trabalhavam em microempresas informais. PMEs são a maior fonte de novos empregos em diversos países subdesenvolvidos (Botswana, Kenya, Malawi, Zimbábue) e eles absorvem mais de $40 \%$ dos novos trabalhadores chegando ao mercado (LIEDHOLM e MEAD, 1999) e têm um significado especial em termos socioeconômicos nas sociedades locais.

$\mathrm{Na}$ Alemanha, onde pequeno é definido como tendo menos que 100 empregados (WEIMER, 1990) a parte dos pequenos negócios na economia como um todo, conforme mostrado no censo de 1970 , atingiu $98,9 \%$ de todas as empresas. A participação das PMEs no emprego não é tão significante, mas mesmo assim atinge $44,2 \%$ de todos os empregados. Informação mais recente (1984) confirma que $71,2 \%$ das empresas no setor manufatureiro são pequenas.

Amadieu (1990) mostra que na França a importância relativa das PMEs está crescendo, e em 1985 as empresas com menos de 500 empregados atingiam $64,5 \%$ da força de trabalho, e $50,9 \%$ do valor adicionado na economia. Duché e Savey (1986), citados por Julien (1998), mostram que regiões com o mais alto nível de crescimento e criação de empregos na França localizam-se exatamente onde a contribuição dos pequenos negócios manufatureiros era a mais alta. O mesmo fenômeno pôde ser observado em algumas partes do Canadá, Inglaterra e Itália (CROSS, 1987; SFORZI, 1989).

Becattini (1990) informa que as grandes empresas - aquelas com mais de 500 empregados - tiveram sua participação na economia italiana reduzida de 25,6\% em 1971 para 18,5 \% em 1981, e no Reino Unido, Marsden (1990) informa que $64,9 \%$ dos trabalhadores eram empregados por PMEs em 1986.

O quadro seguinte dá uma idéia da magnitude dos pequenos e médios negócios: 
Quadro 1 - PARTICIPAÇÃO DAS PMES NA ECONOMIA (PAÍSES SELECIONADOS)

\begin{tabular}{|l|c|c|c|c|}
\hline \multicolumn{1}{|c|}{ País } & Ano & $\begin{array}{c}\text { \% força de } \\
\text { trabalho }\end{array}$ & $\begin{array}{c}\text { \% no. de } \\
\text { empresas }\end{array}$ & \% PIB \\
\hline Estados Unidos & 1996 & 53 & 99,7 & $50-52$ \\
Austrália & 1999 & 45 & 96,9 & - \\
Alemanha & 1984 & 44 & 98,9 & $50-52$ \\
Inglaterra & 1986 & 56 & 99,8 & $50-53$ \\
Brasil & 1994 & 59 & 98,0 & 53 \\
Japão & 1994 & 78 & 99,1 & $52-55$ \\
México & 1995 & 50 & 98,0 & - \\
\hline
\end{tabular}

FONTE: Loveman e Sensenberger, 1990; Sebrae, Brasil: Estudos Sebrae (1994). OECD, Paris: Summary Report (1995); Liedholm e Mead (1999).

\section{A TEORIA DA ECONOMIA DE ESCALA}

O conceito original de economia de escala foi desenvolvido por Adam Smith (1776) e incorporado à teoria neoclássica por Marshal (1920). A teoria é simples como parece: a redução no custo unitário resulta do aumento da produção. Economias de escala emergem quando a firma reduz custos operacionais pela adição de trabalho especializado e volumes adicionais de capital. A firma cresce até que atinge o ponto ótimo ou o mais eficiente tamanho de produção, ou escala (KIRCHHOFF, 1991). Em outras palavras, as firmas que operam neste ponto ótimo terão comparativamente custos mais reduzidos que as suas contrapartidas no mercado que não estão operando numa escala mais eficiente. Então, a teoria mantém que, em um dado mercado, tamanho é o que assegura eficiência (TOMMASO et al., 2000). Conseqüentemente, as PMEs foram consideradas, por definição, ineficientes, um aspecto lembrado anteriormente por Audretsch (1995), que argumenta que a maioria delas opera em níveis sub ótimos.

Entretanto, o mercado demonstrou uma realidade substancialmente diferente: existe uma enorme quantidade de pequenos negócios servindo uma imensa quantidade de usuários, e operando com diversos graus de eficiência. Penrose (1959) tentou explicar a existência e permanência de tantos pequenos negócios e sugeriu uma explicação posteriormente chamada por Julien (1993; 1998) de “teoria dos interstícios" ou, como é mais comumente conhecida, nichos. De fato, muitas PMEs sobrevivem no mercado algumas vezes à sombra de um gigante corporativo, por que são altamente especializadas em atender clientes crescentemente ricos e afluentes, e que deman- 
dam mais variedade e diferenciação, conforme analisado por Veblen (1912) e Scitovsky (1958). As PMEs compensam a ausência de economias de escala por maior flexibilidade, especialmente em tempos de rápidas mudanças (MILLS e SCHUMANN, 1985; CARLSSON, 1989). Isto foi compreendido pelas grandes empresas, que tentam compensar esta situação desfavorável criando pequenas unidades que se comportam como PMEs (CARRÉE et al., 2002) ou, mais simplesmente, utilizando pequenas empresas como subcontratadas em diversos tipos de acordos ou parcerias. ${ }^{4}$ Audretsch (1995, p. 171) relembra que as pequenas empresas tendem a utilizar o fator trabalho mais intensivamente, talvez como uma compensação pela menor escala.

As economias de escala geradas pelas grandes são freqüentemente reduzidas por deseconomias (mais funcionários, instalações, equipamentos, veículos, etc.) inevitáveis para o atendimento de pequenos mercados dispersos geograficamente (TOMMASO et al., 2000), assim permitindo a existência de pequenos competidores, altamente especializados e flexíveis. ${ }^{5}$ Eles não têm as chamadas contra-economias de escala, definidas em vários estudos como custos de capacidade, custos de transformação e custos de ajustamento (GOULD, 1969); custos de coordenação e controle (SCHERER, 1980), custos de informação (SCITOVSKY, 1958) e, mais recentemente, alguns dos custos relacionados com a burocracia, necessidades sociais, e por último, mas não menos importante, o ambiente. Em suma, grandes firmas são submetidas a um grande número de custos de transação (COASE, 1937), os quais estão aumentando substancialmente conforme a economia avança em termos de sofisticação. Entretanto, aparentemente as PMEs são afetadas por custos de transação em menor escala (CARRÉE e THURIK, 2002) porque criam um "transaction space" baseado em confiança e cooperação e que reduz custos e incertezas (JULIEN, 1998, p. 41).

As grandes corporações têm de fazer frente à aversão ao risco demonstrada pelo comportamento de gerentes e líderes de negócios em

${ }^{4}$ No final dos anos 1980, uma das principais montadoras operando no Brasil criou diversas parcerias com PMEs operando dentro de suas próprias fábricas para produzir um automóvel de classe mundial (fabricado simultaneamente em diversas partes do mundo). Todos os parceiros neste projeto são altamente especializados em um ponto específico da linha de produção.

${ }^{5} \mathrm{O}$ mercado de produtos derivados de carne é controlado no sul do Brasil por dois grandes processadores de carne que distribuem uma grande variedade dos seus produtos em cidades médias e grandes. Por causa do cada vez maior custo de distribuição e comercialização em pequenos vilarejos e cidades distantes dos grandes mercados, um número crescente de microempresas e distribuidores, em conexão com produtores locais sintonizados com as necessidades dos clientes, começaram a operar e competir com sucesso com as duas grandes empresas. 
relação à crescente incerteza e volatilidade dos mercados, maior competição e à "destruição criativa"de Schumpeter (1942). ${ }^{6}$ Para reduzir riscos gerados por tais fatores, grandes negócios tentam estabelecer certas formas de controle, como o aumento de tamanho em formas diretas (expansão física, compra de outras fábricas, etc.) ou indiretas (formando cartéis ou adotando posições oligopolísticas no mercado nacional ou internacional). ${ }^{7}$ As PMEs, por sua vez, tentam estabilizar seu limitado ambiente através da cooperação, adaptação ao trabalho, menor nível de especialização e custo de produção (ARZENI, 1997; STEWART e ROTH, 1999; WAGNER e STERNBERG, 2002).

Alguns pesquisadores têm demonstrado que, em diferentes países e considerando o nível relativo de capital envolvido, algo entre 50 e $60 \%$ das PMEs inovam - uma maneira de continuar crescendo - e destas, 5 a 10\% inovam radicalmente (ARCHIBUGGI e CESARETO, 1989; BERNARD e TORRE, 1994) e, apesar de que poucas PMEs conduzem programas de pesquisa e desenvolvimento de maneira sistemática como as grandes fazem, elas parecem fazer isso mais eficientemente que as grandes empresas (JULIEN, 1998). Em geral, a pesquisa feita nas universidades beneficia mais os pequenos negócios (ACS, 1992), e segundo Audretsch (1995, p. 179), trata-se de "key input for generating innovative activity in small enterprises".

Não se trata do desaparecimento dos grandes negócios, ou de que serão drasticamente reduzidos, mas de um novo equilíbrio entre pequenas e grandes empresas. Em alguns mercados, a concentração em grandes empresas parece ser um passo necessário para enfrentar os competidores internacionais, como montadoras de automóveis, instituições financeiras, siderúrgicas, petróleo, etc. (AUDRETSCH, 1995). Ao mesmo tempo, as oportunidades geradas por cooperação e "networking”" (ARZENI, 1997), a criação de novos nichos de mercados determinados por novos hábitos, e os benefícios da complementaridade produtiva (JULIEN, 1998) são fortes sinais de que as PMEs e a economia baseada em milhares de pequenos negócios adaptáveis à mudança e a segmentação de mercado chegaram para ficar.

${ }^{6}$ Schumpeter descreveu o processo de entrar em um mercado utilizando inovação e tomando "market share" dos outros produtores, assim criando riqueza e destruindo as estruturas de mercado existentes. Este processo é a essência do desenvolvimento econômico (HEBERT e LINK, 1989) e é chamado de "destruição criativa" (KIRCHHOFF, 1991).

${ }^{7}$ Duas grandes multinacionais (Ford e Volkswagen) decidiram operar sob um único nome no Brasil, nos primeiros anos da década passada. Ambas controlaram, por um tempo, mais de $50 \%$ do mercado. Entretanto, não puderam administrar as diferenças em termos culturais e administrativos, e os problemas de escala. Esta situação levou a uma ruptura, e ambas as companhias perderam participação no mercado. 


\section{MERCADO DE TRABALHO}

Qual o significado de pequeno na área dos negócios? Podem ser uma surpresa as muitas definições estatísticas de tamanho e heterogeneidade da firma. Potobsky (1992) menciona que existem mais de 50 definições de PMEs em 75 países diferentes. Existem algumas distinções mínimas dentro dos setores primário e de serviços, e mais distinções no setor manufatureiro. Diversos autores tentam definir tipologias, por tipo de objetivos administrativos, estratégia e potencial da empresa (CARLAND et al., 1984; MARCHESNAY, 1988; MARCHINI, 1988, e outros); alguns autores definem tipologias por organização e crescimento (WEBSTER, 1976; HOSMER et al., 1977; VARGAS, 1984; e outros). Finalmente, tipologias por setor ou tipo de mercado encontraram defensores em Preston (1977), Vésper (1979), Candau (1981), Potier (1986) e Julien (1993). A mais simples classificação é por tamanho: volume de vendas, nível de capital ou, na maioria dos casos, número de empregados (JULIEN, 1998, p. 7). Este critério é adotado em quase todos os países:

Quadro 2 - TAMANHO DA FIRMA POR NÚMERO DE EMPREGADOS: UM RESUMO

\begin{tabular}{|lc|c|c|c|c|}
\hline & Micro & Muito Pequena & Pequena & Média & Grande \\
\hline $\begin{array}{l}\text { No. de } \\
\text { Empregados }\end{array}$ & 1 a 4 & 5 a 19 & 20 a 99 & 100 a 499 & +500 \\
\hline
\end{tabular}

FONTE: OECD, "Globalization of Economic Activities and the Development of SMEs". Paris: Summary Report, 1995.

O quadro seguinte dá uma idéia da participação das PMEs no nível de emprego geral de alguns países selecionados (desenvolvidos): 
Quadro 3 - NÍVEL DE EMPREGO POR TAMANHO (NÚMERO DE EMPREGADOS)

\begin{tabular}{|l|c|c|c|c|c|}
\hline \multicolumn{1}{|c}{ País } & Ano & $\mathbf{- 2 0}$ & $\mathbf{2 1 - 9 9}$ & $\mathbf{1 0 0 - 4 9 9}$ & $\mathbf{5 0 0 +}$ \\
\hline Estados Unidos & 1982 & & 46 & 13 & 41 \\
Japão & 1985 & $38(1)$ & $18(2)$ & 17 & 27 \\
França & 1985 & 26 & 20 & 18 & 36 \\
Alemanha & 1970 & $17(3)$ & $24(4)$ & 18 & 41 \\
Noruega & 1985 & $47(5)$ & $10(6)$ & $10(7)$ & $33(8)$ \\
Suíça & 1985 & & $46(9)$ & $27(10)$ & 27 \\
Itália & 1981 & 53 & 16 & 12 & 19 \\
\hline
\end{tabular}

1) 1-29 empregados; 2) 30-99 empregados; 3) 1-10 empregados; 4) 10-100 empregados

5) 0-49 empregados; 6) 50-99 empregados; 7) 100-199 empregados; 8) 200+ empregados

9) 1-49 empregados; 10) 50-499 empregados.

FONTE: informações oriundas de relatórios contidos em informativos de diversos países, compiladas por Loveman et al. (1990).

A participação dos pequenos negócios no emprego geral mostrou uma tendência negativa do final do século 18 até meados da década de 1970 (CARRÉE et al., 2002). Na Alemanha, pequenas empresas (com menos de 50 empregados) tiveram sua participação reduzida de $78 \%$, em 1882, para $57 \%$ em 1950, e 44\% em 1970; nos Estados Unidos, micro e pequenas empresas (menos de 100 empregados) caíram de 52\% em 1909 para 32\% em 1947, e para 31\% em 1977 (LOVEMAN et al., 1990).

Entretanto, esta tendência histórica foi revertida em muitos países: novos empreendimentos estavam crescendo depois da metade dos anos 1970 e, apesar da recessão mundial depois do choque de petróleo em 1973 e novamente no começo dos anos 1980, este crescimento foi mantido. A Dinamarca criou a maior parte dos novos negócios, em bases relativas, seguida pela Bélgica, Alemanha, Inglaterra, Espanha e Portugal. A mais baixa taxa de crescimento de novos negócios foi observada nos Estados Unidos, seguida pela da Holanda, Itália e França (JULIEN, 1998).

Em um trabalho desenvolvido por Baldwin e Picot (1994), o qual inclui dados do Canadá e dos Estados Unidos, constatou-se que a maior parte da destruição de empregos é feita por grandes empresas (mais de 500 empregados). A mudança líquida, positiva, em termos de "job turnover" (criação de empregos menos destruição de empregos) foi feita por pequenos negócios no período 1970-1988.

$\mathrm{O}$ fato de que as PMEs são agora reconhecidas como as principais geradoras de novos empregos (POMBO e HERRERO, 2001) não é restrito aos Estados Unidos, e pode ser verificado na vasta maioria do mundo 
industrializado, apesar de que as discussões geradas demonstraram que a maioria dos estudos (incluindo o de Birch) sobre o processo de geração de empregos foi prejudicada (LOVEMAN et al., 1990) por problemas de amostragem, representação setorial, fechamento de empresas, etc. A grande massa de críticas sobre este assunto não elimina o fato de que as PMEs desempenham um papel crucial no mercado de trabalho. A esse respeito, algumas importantes conclusões foram comentadas e criticadas por Dennis, Philips e Starr (1994, p. 23), como segue:

1. PMEs são responsáveis pela criação de mais de dois terços das novas posições de trabalho no setor privado, nos últimos vinte e cinco anos nos Estados Unidos.

2. As muito pequenas (micro) empresas (aquelas com 1-19 empregados) produziram a maioria dos novos empregos relativamente à sua participação no emprego total.

3. Empregos perdidos nos pequenos negócios são primariamente uma função da liquidação (encerramento) de negócios em vez de contração de negócios, ao contrário das grandes empresas, que aparentam ser mais relacionadas com ciclos de negócios.

4. As PMEs estão se tornando cada vez mais importantes no setor manufatureiro, justamente o oposto da crença de que o setor manufatureiro seria o reino das grandes companhias.

5. As pequenas empresas não somente criam a maioria líquida dos novos empregos como também criam a maioria dos novos empregos e destroem a maioria dos velhos empregos.

Brock et al. (1989) argumenta que as pequenas empresas pagam menores salários (uma conclusão a que chegaram RATTNER, 1985, no Brasil; LOVEMAN et al., 1990; e AUDRETSCH, 1995), mesmo considerando algumas características dos trabalhadores (experiência, educação), se a firma é sindicalizada ou não, e o ramo de negócio. Adicionalmente, PMEs são mais propensas a trabalho temporário ou parcial. Prado (1995, p. 25) argumenta que, no Brasil, "a maioria das empresas informais não oferece as menores condições de trabalho...". Acs (1992, p. 41) argumenta que elas oferecem empregos de baixa qualidade, e os trabalhadores são "less likely to receive health insurance, pension and other fringe benefits, or training". Davis et al. (1994) discutem o conceito de qualidade do trabalho, também argumentando que a simples criação de postos de trabalho não é um objetivo apropriado da política econômica, adicionando a conclusão de que no setor manufatureiro americano grandes empregadores ainda desempenham um papel dominante na geração de empregos, apesar da taxa mais alta de criação (e destruição) de empregos entre pequenos empregadores. Dennis 
et al. (1994, p. 27) advogam que qualidade do emprego é uma questão isolada, específica, e os estudos devem concentrar-se na questão da dinâmica da geração de empregos.

Davis et al. (1994, p. 18), em suas conclusões, argumentam que tratamento preferencial na área fiscal ou de crédito direcionada às PMEs falha em compreender como o número (ou a qualidade) dos empregos responde a uma mudança política. Com ceticismo, concluem que a determinação de como tal mudança afeta o número de empregos é um desafio considerável, porque as propostas implicam em conflitos políticos a respeito da estrutura de subsídios, impostos, e o próprio tratamento regulatório; argumentam, ainda, que tais conflitos são caros, em termos sociais, e refletem principalmente a força política das partes, mais do que o simples critério econômico.

Brock et al. (1989), com mais moderação, concluem que os programas de assistência aos pequenos negócios são justificáveis, porque PMEs estão mais sujeitas a problemas de liquidez, e assim, a assistência governamental pode aumentar a eficiência econômica. ${ }^{8}$ Mais ainda, pequenos negócios empregam trabalhadores de baixa especialização, ao contrário das grandes empresas, sendo então concebível que o governo produza, por exemplo, um programa que isente as PMEs de alguns custos fixos com o objetivo de aumentar a absorção de trabalhadores desempregados. Asquith e Weston (1994, p. 4) concluem que o processo da criação de empregos continuará a ser desempenhado pelas pequenas empresas, e a regulamentação não deve sobrecarregá-las.

\section{FATORES FINANCEIROS E DE GESTÃO}

Problemas financeiros afetam firmas de todos os tamanhos (JULIEN, 1998). Entretanto, por causa do paradigma de que somente gran-

${ }^{8}$ No Brasil, os esforços para controlar a taxa inflacionária quase sempre incluíram contingenciamento de crédito por meio das instituições financeiras, esquecendo das diferenças existentes entre os grandes e pequenos negócios. O resultado não foi bom para as PMEs, que sentiram profundamente os efeitos das restrições de crédito e de capital. Esta situação mudou parcialmente nos últimos anos com a difusão do microcrédito, que beneficia as microempresas e os auto-empregados (sobre sua importância, ver FAIRLEY, 1998; LIEDHOLM et al., 1999, p. 107), e também crédito tornado disponível por bancos oficiais. Em junho de 2003 o governo brasileiro lançou um programa de estimulo ao microcrédito, cercado de ceticismo quanto à sua eficácia (ver revista Pequenas Empresas, Grandes Negócios, edição de agosto de 2003, p. 9-10). 
des empresas são eficientes e têm condições de sobreviver no longo prazo, o governo e instituições financeiras têm prestado pouca atenção aos pequenos negócios. Esta situação (falta de suporte creditício) tende a ser amplificada por outros fatores, identificados por Berryman (1982) e mencionados por Julien (1998, p. 276); os dois últimos são freqüentemente mencionados como os mais importantes quando se consideram razões para o fracasso:

a. Dependência do proprietário / gerente;

b. Dificuldade de delegar autoridade;

c. Por causa da forte competição, o poder de mercado é limitado;

d. Problemas com fluxo de caixa e conseqüente vulnerabilidade;

e. Falta de habilidades gerenciais;

f. Falha em revisar o desempenho financeiro, inexistência de planejamento financeiro e um plano de negócios.

Tamari (1980, p. 20) afirma que "pequenas empresas têm acesso limitado ao capital e aos mercados de dinheiro e assim sofrem de crônica descapitalização. Como resultado, elas são mais propensas a recorrer a capitais mais caros que atuam como um freio no seu desenvolvimento econômico". 9 Pombo e Herrero (2001, p. 76) argumentam que "los bancos ponen especial énfasis en la garantia y com frecuencia niegam el aceso al credito a largo plazo". Lessa (2004, p. A11) menciona que as PMEs são de "difícil bancabilidade". Bancos comerciais e de investimento no Brasil estão habituados a emprestar dinheiro de fundos oficiais (distribuídos por entidades do governo federal) para as grandes companhias, que, teoricamente, representam um risco de crédito melhor. Elas não requerem uma análise de crédito muito rigorosa, permitindo assim que os bancos cobrem uma taxa de juros menor. Conseqüentemente, as PMEs têm de utilizar empréstimos (mais caros) de curto prazo (RATTNER, 1985; SEBRAE, 1995, p. 16). É uma situação comum (e desafiadora) para as PMEs e microempresas fazer face a contratos bancários sofisticados em que existem diferentes períodos para cálculo de juros, taxas e comissões adicionais, combinadas com outras práticas financeiras, como a exigência de garantias em forma de recebíveis (que são mantidas numa conta separada sem cálculo de juros). Soluções alternativas, como a troca de empréstimo de curto prazo por outros menos caros e de longo prazo, ou então redução de estoque, tentando manter um balanço adequado entre os pagamentos a fornecedores e os recebíveis, são colocadas em prática por poucos proprietários/gerentes, assim aumentando o risco de problemas ou fracasso (BORTOLLI NETO, 1994, p. 10).

\footnotetext{
${ }^{9}$ Tradução do autor.
} 
Julien (1998) argumenta que existe um gap financeiro no fornecimento de crédito para as pequenas empresas, que as inibe de crescer e amadurecer, um ponto anteriormente lembrado por Edwards (1994). Este gap financeiro tem dois componentes principais, que são o uso limitado de fundos disponíveis por causa da falta de conhecimento e expertise para entendêlos e utilizá-los; e segundo, fundos escassos são distribuídos entre os melhores riscos pelas instituições financeiras, que entendem que os melhores são os maiores. Jefferson (1997, p. 1), analisando pequenos negócios e seus constrangimentos financeiros, conclui que "it is an established fact that small firms do not have direct access to financial markets", e Edwards (1994, p. 3) menciona a sua "inability to raise finance."

O mercado financeiro é tradicionalmente desfavorável às PMEs. A reação à (fraca, desconhecida) nova e pequena empresa é quase sempre negativa. As dificuldades para obter crédito, especialmente de longo prazo, são enormes. Esta situação faz com que proprietários/gerentes manifestem uma clara preferência para empréstimos em relação a aumentos de capital. Holmes e Kent (1991) notam que proprietários/gerentes de pequenas empresas não desejam compartilhar o controle de suas empresas e preferem um alto grau de independência sem empréstimos e suas cláusulas restritivas. Anteriormente, Robinson e Hunt (1988) notam que autonomia é uma grande força motivacional para pequenos empresários.

Apesar de alguns autores terem concluído que pequenos negócios têm mais ativos líquidos do que os grandes, como discutido por Julien (1998, p. 285), outros chegaram à conclusão oposta. Algumas PMEs são "líquidas" no sentido de que dispõem de um volume reduzido de ativos fixos e um grande volume de recebíveis, que podem ser convertidos rapidamente em caixa; outras dependem de estoque e de financiar clientes por um tempo mais longo do que outras. Entretanto, a conclusão de que todas as PMEs têm problemas com seu fluxo de caixa e seu capital de giro é de alguma forma estereotipada. A pesquisa de problemas financeiros como estes tem de considerar todas as diferentes características que são dependentes do estágio de desenvolvimento, tamanho, setor, nível de lucratividade, o mercado, as informações financeiras disponíveis, e assim por diante. Calof (1985) argumenta que a sofisticação de algumas estruturas financeiras, numa pesquisa feita com 115 PMEs no Canadá, aumenta em proporção às necessidades da firma e do nível de conhecimento do proprietário/gerente. Em muitos casos, por causa da falta de expertise na área administrativa e financeira por parte do proprietário/gerente e ausência de uma estrutura formal de planejamento (FRIZELLE, 2001), as PMEs são deixadas sem opções e têm de aceitar esquemas caros de contratos bancários ou tentar sobreviver com seu próprio capital de giro. 
Larson e Clute (1979) listam alguns fatores financeiros e características pessoais de proprietários/gerentes de pequenos negócios que os fazem propensos a problemas financeiros, como pode ser visto no quadro a seguir:

\section{Quadro 4 - ATRIBUTOS PESSOAIS DE PROPRIETÁRIOS / GERENTES EM PMES FALIDAS}

\begin{tabular}{|c|c|c|}
\hline $\begin{array}{l}\text { Atributos } \\
\text { Pessoais }\end{array}$ & $\begin{array}{l}\text { Deficiências } \\
\text { Gerenciais }\end{array}$ & $\begin{array}{c}\text { Fatores } \\
\text { Financeiros }\end{array}$ \\
\hline $\begin{array}{l}\text { 1. Tendência a superestimar } \\
\text { o próprio conhecimento } \\
\text { gerencial. } \\
\text { 2. Baixo nível educacional. } \\
\text { 3. Inflexibilidade e } \\
\text { resistência à mudança. } \\
\text { 4. Uso da opinião pessoal } \\
\text { como padrão. } \\
\text { 5. Processo decisório } \\
\text { baseado em intuição e } \\
\text { emoção. } \\
\text { 6. Orientação para o } \\
\text { passado e não futuro. } \\
\text { 7. Pouco contato com o } \\
\text { desenvolvimento de seu } \\
\text { ramo de negócios. } \\
\text { 8. Resistência a } \\
\text { sugestões de pessoal } \\
\text { qualificado. }\end{array}$ & $\begin{array}{l}\text { 1. Fraca e / ou inexistente identifi- } \\
\text { cação de mercado ou zona comer- } \\
\text { cial. } \\
\text { 2. Inexistência de técnicas objetivas } \\
\text { para localização. } \\
\text { 3. Falha no entendimento dos } \\
\text { princípios da delegação. } \\
\text { 4. Considera propaganda como } \\
\text { despesa, não investimento. } \\
\text { 5. Inexistência de planejamento } \\
\text { efetivo. } \\
\text { 6. Conhecimento rudimentar de } \\
\text { precificação, estratégias e táticas. } \\
\text { 7. Falha em entender o princípio } \\
\text { dos canais de distribuição. } \\
\text { 8. Falha em motivar empregados. } \\
\text { 9. Crença que os problemas são } \\
\text { exógenos. }\end{array}$ & $\begin{array}{l}\text { 1. Frágeis controles de estoque. } \\
\text { 2. Contabilidade incorreta e } \\
\text { livros incompletos. } \\
\text { 3. Falha no entendimento da } \\
\text { importância do fluxo de caixa e } \\
\text { liquidez. } \\
\text { 4. Inabilidade para conferir a } \\
\text { acuidade da contabilidade. } \\
\text { 5. Falha em utilizar números } \\
\text { financeiros para planejar o } \\
\text { futuro. } \\
\text { 6. Inexistência de controles de } \\
\text { entrada e saída de fundos. } \\
\text { 7. Falha em entender a } \\
\text { terminologia contábil. }\end{array}$ \\
\hline
\end{tabular}

FONTE: Sumário preparado por Julien (1998) com base no trabalho de Larson e Clute (1979).

A dificuldade de interpretar informações é compreensível porque o planejamento formal é normalmente inexistente (MARCHESNAY, 1988); deste modo, esforços de marketing não são adequadamente coordenados ou são desempenhados de uma maneira deficiente. ${ }^{10}$ Adicionalmente, as PMEs apresentam desempenhos diferentes no mercado por causa de ques-

${ }^{10}$ No Brasil, na segunda metade da década passada, houve a criação de muitos pequenos negócios vendendo artigos importados por R \$ 0,99, e lojas de aluguel de vídeos. Em muitos casos, lojas foram abertas em frente a outra porque a região parecia "boa" para isso, ou então o competidor que havia chegado primeiro "estava ficando rico". Apesar de alguns esforços feitos por uma agência de treinamento governamental, nenhuma pesquisa de mercado ou plano de negócio foi utilizado por estes microempresários/empreendedores, que tiveram de fazer face aos resultados da concentração algum tempo depois e, em muitos casos, fecharam suas portas. 
tões de escala e dimensões físicas; limitada presença e imagem pública fraca; pequena participação de mercado e falta de lealdade dos consumidores; tempo limitado para gerência e falta de gerentes profissionais, e uma mistura de objetivos pessoais e financeiros que freqüentemente reduz e onera o caixa da empresa (HILLS, 1987; FONSECA, 2004).

A literatura financeira para as PMEs não apresenta uma rigorosa análise estatística; as evidências para outros países que não os Estados Unidos são quase inexistentes (MCMAHON e HOLMES, 1991). Nayak e Greenfield (1994), citados por Gorton (1999), revelam que, de uma amostragem analisada no Reino Unido, somente $34 \%$ das empresas usam alguma forma de planejamento financeiro (como orçamento), e 16\% não mantêm controles de suas dívidas. Mais recentemente, Gorton (1999) encontrou evidências de que em torno de 50\% de uma amostragem de 366 PMEs no Reino Unido utilizavam alguma forma de planejamento financeiro; $25 \%$ faziam pesquisa de mercado. Frizelle (2001, p. 6) descobriu que 53\% de uma amostragem composta por 27 SMEs na Inglaterra estão dispostos a fazer planejamento financeiro quando atingidos por uma grande mudança ou crise. Fuller (1994, p. 34) afirma, "planning is a problem for small firm management which tends to be reactive...". As companhias que não utilizam planejamento não conseguem obter financiamento adequado e, em vez disso, têm de utilizar o suporte da família ou amigos, ou mesmo qualquer forma de capital pessoal (GORTON, 1999).

Em pesquisa efetuada com uma amostragem de empresas brasileiras, Bortolli Neto (1994, p. 8) constata que $90 \%$ da amostragem do setor industrial apresentavam problemas estratégicos; do setor comercial, 79\%, e o setor de serviços em geral, 70\%. O autor considera que os "problemas empresariais que afetam as PMEs ... são principalmente os de natureza estratégica."

Em um estudo desenvolvido por Ellis e Jolibert (1991), com um grupo de 106 pequenas e médias empresas no Brasil, no período de 1976 a 1986, uma correlação foi constatada entre a taxa de sobrevivência das PMEs e o conhecimento da concepção, atitudes e atividades de marketing. Esta correlação parece ser mais forte para empresas médias do que empresas pequenas. $\mathrm{O}$ estudo dividiu um grupo de PMEs em sobreviventes e nãosobreviventes e concluiu-se que todos os sobreviventes mudaram sua linha de produção; o uso de propaganda pelos sobreviventes atingiu a taxa de 2,7 contra 1,2 para os não sobreviventes; a vasta maioria das firmas fixou seus preços na base tradicional de custos e benefícios, sem considerar os efeitos da competição. Nenhuma diferença significante foi encontrada entre sobreviventes e não sobreviventes relacionada com canais de distribuição. Entre- 
tanto, atitudes gerenciais mostraram diferenças significantes em favor dos sobreviventes, os quais fizeram mais mudanças em seus produtos, maior uso de publicidade diversificada e promoções, usaram uma rede múltipla de distribuição e foram influenciados pela competição para fixar preços. Os autores notaram que a prática de marketing teve um impacto significante na sobrevivência de empresas de um país em desenvolvimento, onde supostamente o uso do planejamento de marketing como uma ferramenta é deficiente.

Planejamento é uma área claramente negligenciada por proprietários/gerentes de pequenos e médios negócios, e empreendedores. Em parte, por causa de atitudes e treinamento inadequados, dificuldades com custos e despesas, e nenhuma margem para manipular preço e melhorar a lucratividade, conforme mostrado por Larson e Clute (1979). Alguns autores (JULIEN, 1998) mostraram que as PMEs têm menos espaço para manobrar nesta área em comparação com empresas de grande porte, e freqüentemente têm medo de perder clientes quando mudando preços em seus nichos de mercado particulares. Downing (1971) sugere que, mesmo garantindo sua permanência no mercado, o planejamento e as estratégias de marketing empregadas pelas PMEs não são tão claros quanto nas grandes empresas.

\section{CONCLUSÃO}

Economia dos Pequenos Negócios é uma área que, de acordo com o superado paradigma da escala, requer apenas alguma espécie de treinamento, já que não é considerada uma verdadeira disciplina acadêmica. A resistência para implementar cursos nesta área é notória e a área do empreendedorismo sofre com o mesmo dilema. Carneiro (1994, p. 22) afirma que "a pequena empresa (no Brasil) só era prioridade para um punhado de estudiosos". Esta situação (falta de credibilidade acadêmica) não é privilégio de nenhuma região e a preocupação com este assunto pode ser observada em muitas partes do mundo. Houve considerável progresso, e esforços feitos por alguns países produziram bons resultados; a produção intelectual está crescendo com alguns centros de excelência de relevante produção científica. Nos Estados Unidos, de pouco mais de uma dúzia de cursos em pequenos negócios/empreendedorismo nos anos 1960, existem hoje mais de 1600. No Brasil, o progresso não é tão rápido quanto se poderia desejar, mas pode ser constatado em diversas universidades e outras instituições de ensino. 
É obviamente difícil definir uma teoria para pequenos negócios porque as PMEs se compõem de elementos fundamentalmente diferentes. De fato, pequenos negócios como entidade não existem; há tantos tipos diferentes de empresas, e os comportamentos e atitudes dos proprietários/ gerentes são muito diferentes daqueles mostrados nas grandes corporações. Adicionalmente, o ambiente físico desempenha um papel importante definindo características das pequenas empresas. Um status diferente é reservado para a grande empresa, a qual, por definição, é capaz de atuar no ambiente físico com grande efetividade e força, mudar as preferências do seu mercado, impondo preços e produtos por meio de campanhas maciças, criar novas conexões com regiões ou países distantes, e eliminar a competição criando cartéis e monopólios.

Destarte, uma nova teoria para os pequenos negócios tem de ser diferente das teorias tradicionais de economia e/ou administração usualmente aplicáveis aos grandes negócios. Julien (1988, p. 403) argumenta que é necessário "invent a new theory that takes account of the systematic creation and development of small firms, and the subsequent disappearance of many of them - in other words, a theory of instability." É importante tentar reduzir a diferença entre a firma na teoria e a firma no mundo real (TOMMASO et al., 2000), considerando sua heterogeneidade e sua característica de construir relacionamentos mais do que competição (apesar das dificuldades apontadas em SEBRAE, 1994, p. 45), e sua amigável relação com o ambiente - que desempenha mais e mais um papel decisivo nas preocupações da humanidade neste século - e com o qual as PMEs vivem numa espécie de simbiose (JULIEN, 1998).

Kirchhoff (1991, p. 109) argumenta sobre o conflito entre a visão macroeconômica que prejudica os pequenos negócios e o empreendedorismo, e advoga uma nova teoria econômica porque "the absence of a widely held theory of entrepreneurship constrains not only economics but also all of the disciplines that extend their interests into the entrepreneurship are$n a$ ". Considerando o papel preponderante das pequenas e empreendedoras empresas na área da inovação, a força básica da economia (SCHUMPETER, 1942), e o fato de que, em algum momento do processo criativo, todo empreendedor é também um proprietário/gerente de alguma PME (e vice-versa), é também aceitável estender este argumento para os pequenos negócios de um modo geral (CARRIER, 1996).

O tamanho não é uma variável absoluta para identificar a habilidade da empresa para a competição e sobrevivência no mercado, nem uma condição para a criação de políticas macroeconômicas que equivocadamente beneficiam as grandes corporações em detrimento das pequenas. A des- 
peito do crescente conhecimento sobre o impacto socioeconômico de novas políticas direcionadas aos pequenos negócios no mundo, existe

a dangerous space between the evident spreading of policies aimed at small firms and the lack of a unitary theoretical structure capable of verifying (both ex ante and ex post) the feasibility, sustainability and coherence of these policies (TOMMASO et al., 2000, p. 41).

Analisando os extensos programas do Banco Mundial mantidos como apoio às PMEs em várias partes do mundo com o objetivo de reduzir o desemprego e a pobreza, Beck et al. (2005), utilizando uma amostragem de 45 países, não encontraram suporte empírico à idéia de que políticas oficiais devem oferecer subsídios às pequenas e médias empresas, apesar da associação positiva entre a importância econômica das PMEs e o PIB per capita. As discussões de Kirchhoff (1991) sobre as fraquezas da teoria neoclássica do equilíbrio geral também adicionam mais preocupações sobre a falta de um corpo analítico de conceitos, para ajudar a criar um guia consistente e coerente para o desafio de estabelecer políticas econômicas.

Quaisquer que sejam os resultados produzidos pelos esforços para evoluir da teoria neoclássica para uma visão moderna da importância, relevância, e contribuição dos pequenos negócios para o progresso econômico (ACS et al., 1998), é importante enfatizar que os resultados da pesquisa e contribuição acadêmica devem considerar as diferenças entre as economias do mundo industrializado e a necessidade de "transposing what emerges from this literature to policy interventions destined for less economically developed situations" (TOMMASO et al., 2000, p. 42). Em outras palavras, aquilo que é uma política adequada para países desenvolvidos pode ser transformada em um equívoco se aplicada para alguns países da América Latina, Leste Europeu, ou alguns países asiáticos, onde os hábitos, cultura, e o estágio econômico diferem significantemente. Um esforço adicional será necessário antes que economistas e responsáveis pela política econômica concluam que programas assistenciais para PMEs são eficientes. O simples estímulo a bancos e outras instituições financeiras para distribuir fundos entre as PMEs pode representar uma medida artificial sem conseqüências diretas. Isso pode direcionar de maneira errônea o fluxo de recursos financeiros, sendo assim prejudicial ao processo geral de criação de emprego e renda. 


\title{
RESUMO
}

O objetivo deste artigo é estabelecer uma discussão, com base na literatura existente, a respeito da importância das pequenas e médias empresas (PMEs) a nível macroeconômico, das questões relativas à economia de escala, do nível do emprego, e dos principais problemas financeiros e de gestão que afetam os pequenos negócios de um modo geral, concluindo pela necessidade de desenvolver uma nova e consistente teoria microeconômica que ajude na criação e aplicação de políticas oficiais que fomentem o progresso econômico.

Palavras-chave: pequenos negócios; escala; mercado de trabalho; finanças.

\begin{abstract}
The purpose of this article is to establish a discussion based on the existent literature about the importance of small and medium-sized enterprises (SMEs) at macroeconomic level, about questions related to the economy of scale, the level of employment, and the main financial and managerial problems which affect small businesses in general, concluding with the need to develop a new and consistent microeconomic theory which helps create and apply official policies fostering economic progress.

Key-words: small businesses; scale; job market; finance.
\end{abstract}

\section{REFERÊNCIAS}

ACS, Z. Small business economics: a global perspective. Challenge, v. 35, n. 6, p. 38-44, 1992.

CARLSSON, B.; KARLSSON, C. Entrepreneurship, small and medium-sized enterprises, and the macroeconomy. New York: Cambridge University Press, 1998.

AMADIEU, J. F. France (Capítulo). In: SENGENBERGER, W.; LOVEMAN, G.; PIORE, M. J. (Eds.). The re-emergence of small enterprises: industrial re-structuring in industrialised countries. Geneve: Institut International d'Etudes Sociales, 1990.

ARCHIBUGGI, D.; CESARETTO, S. Piccola impresa e cambriatorento technologico. Modelli teorici e resultate dall'Indagina CNR-STAT sull'innovazione tecnologica nel settore manufatturiero italiano. Piccola Impresa, v. 2, p. 45-73, 1989.

ARRIGHETTI, A.; VIVARELLI, M. The role of innovation in the postentry performance of new small firms: evidence from Italy. Southern Economic Journal, v. 65, n. 4, p. 927-939, 1999. 
ARZENI, S. Entrepreneurship and local development. OECD Observer, v. 204, p. $27-$ 29, 1997.

ASQUITH, D.; WESTON, F. J. Small business, growth patterns, and jobs. Business Economics, v. 29, n. 3, p. 31-34, 1994.

AUDRESTCH, D. B. The role of small-and-medium sized enterprises in $R \& D$, innovation and diffusion of technology. Paper presented at the high-level Small Business Seminar, OECD. Paris, Jul. 1990.

. Innovation and industry evolution. Cambridge, MA: MIT Press, 1995.

BALDWIN, J.; PICOT, G. Employment generation by small producers in the Canadian manufacturing sector. Small Business Economics, p. 324, 1994.

BECATTINI, G. Italy (Capítulo). In: SENGENBERGER, W.; LOVEMAN, G.; PIORE, M. J. (Eds.). The re-emergence of small enterprises: industrial re-structuring in industrialized countries. Geneve: Institut International d'Etudes Sociales, 1990.

BECK, T., KUNT, A. D.; LEVINE, R. SMEs, growth, and poverty. Working Paper 11224, World Bank, Washington, DC. mar. 2005. Disponível em: <http://www.nber.org/ paper/w11224>. Acesso em: 15 set. 2005.

BERNARD, J.; TORRE, A. La dynamique de l'innovation et de R-D des PMI Françaises. Revue Internationale PME, v. 7, n. 3-4, p. 19-41, 1994.

BERRYMAN, J. Small business failure and bankruptcy: a survey on the literature. European Small Business Journal, v. 1, n. 4, p. 47-59, 1982.

$\mathrm{BIRCH}, \mathrm{D}$. V. The job generation process. Final report to economic development administration, MIT. Cambridge, MA: MIT Press, 1979.

BORTOLI NETO, A. Principais problemas das micro e pequenas empresas. Estudos Sebrae, n. 1, p. 8-13, 1994.

BROCK, W. A.; EVANS, D. S. Small business economics. Small Business Economics, v. 1, p. 7-20, 1989.

CALOF, J. Analysis of small business owner's financial preferences. Journal of Small Business and Entrepreneurship, v. 3, n. 3, p. 39-44, 1985.

CANDAU, P. Pour une taxonomie de l'hypofirme. Revue de l'Economie Industrielle, n. $16,1981$.

CARLAND, J. W.; HOY, F.; BOULTON, W. R.; CARLAND, J. A. Differentiating entrepreneurs from small business owners: a conceptualization. Academy of Management Review, v. 9, p. 354-359, 1984.

CARLSSON, B. The evolution of manufacturing technology and its impact on industrial structure: an international study. Small Business Economics, v. 1, n. 1, p. 21-38, 1989.

CARNEIRO, J. G. P. A microempresa 10 anos depois. Estudos Sebrae, ano 1, n.3, p. 21 23, 1994.

CARRÉE, M. A.; THURIK, A. R. The impact of entrepreneurship on economic growth. Centre for Advanced Small Business Economics (CASBEC). Rotterdam: Erasmus University, 2002. Disponível em: <http://www.few.eur.nl/few/people/thurik/publications>. Acesso em: 20 nov. 2005. 
CARRIER, C. Intrapreneurship in small businesses: an exploratory study. Entrepreneurship Theory and Practice, v. 21, n. 1, p. 5-20, 1996.

COASE, R. The nature of the firm. Economica, v. 4, p. 386-405, 1937.

CROSS, M. New firm formation and regional development. Guilford: Gower, Inc., 1987.

DAVIS, S. J.; HALTIWANGER, J.; SCHUH, J. Small business and job creation: dissecting the myth and reassessing the facts. Business Economics, v. 29, n. 3, p. 13-21, 1994.

DENNIS Jr., W. J.; PHILIPS, B. D.; STARR, E. Small business job creation: the findings and their critics. Business Economics, v. 29, n. 3, p. 23-30, 1994.

DOWNING, G. Basic marketing, a strategic systems approach. NY: Merril Publishing Co., 1971.

DUCHÉ, G.; SAVEY, S. Le role de la PME dans la mutation du mode de production capitaliste. In: JULIEN, P. A.; CHICHA, J.; JOYAL, A. (Eds.). La PME dans un monde en mutation. Quebec: Presses de l'Universite du Quebec, 1986.

EDWARDS, P. Finance for small and medium-sized enterprises. International Journal of Bank Marketing, v. 12, n. 6, p. 3-9, 1994.

ELLIS, D. S.; JOLIBERT, A. J. P. The role of marketing in the survival of small firms in a developing region. In: HILLS, G. E.; LAFORGE, R. W. (Eds.) Research at the marketing / entrepreneurship interface. Proceedings of the University of Illinois at Chicago Symposium on Marketing and Entrepreneurship, 1991.

FAIRLEY, J. New strategies for microenterprise development. Journal of International Affairs, v. 52, n. 1, p. 339-350, 1998.

FONSECA, A. M. De um cofre para outro. Pequenas Empresas, Grandes Negócios, p. 98-99, mar. 2004.

FRIZELLE, G. D. M. Business strategy - do SMEs face special problems? Proceedings of The Fourth SMES International Conference. Cambridge, England, 2001.

FULLER, P. B. Assessing marketing in small and medium-sized enterprises. European Journal of Marketing, v. 28, n. 12, p. 34-49, 1994.

GORTON, M. Use of financial management techniques in the U.K. - Based small and medium sized enterprises: empirical research findings. Journal of Financial Management \& Analysis, v. 12, n. 1, p. 56-64, 1999.

GOULD, J. P. Adjustment cost in the theory of investment of the firm. Review of Economic Studies, v. 36, n. 1, p. 31-42, 1969.

GRANT, A. Entrepreneurship - The major academic discipline for the business education curriculum for the $21^{\text {st. }}$. Century. International Small Business Journal, v. 15, n. 3, p. 28-37, 1998.

HEBERT, R. F.; LINK, A. N. In search of the meaning of entrepreneurship. Small Business Economics, v. 1, p. 39-49, 1989.

HILLS, G. E. Marketing and entrepreneurship issues: scholarly justification. Proceedings of Research Symposium on the Marketing/Entrepreneurship Interface. Chicago, 1987. 
HOLMES, S.; KENT, P. An empirical analysis of the financial structure of small and large Australian enterprises. The Journal of Small Business Finance, v. 1, n. 2, p. 141154, 1991.

HOSMER, L.; COOPER, A.; VESPER, K. The entrepreneurial function. Englewood Cliffs: Prentice Hall, 1977.

JEFFERSON, P. N. Unemployment and financial constraints faced by small firms. Economic Inquiry, v. 35, n. 1, p. 108-119, 1997.

JULIEN, P. A. Small business as a research subject: some reflections on knowledge of small businesses and its effects on economic theory. Small Business Economics, v. 5, p. 157-166, 1993.

. The state of the art in small business and entrepreneurship. England: Ashgate Publishing Ltd., 1998.

KIRCHHOFF, B. A. Entrepreneurship's contribution to economics. Conflict between macro and microeconomics with regard to general equilibrium theory. Entrepreneurship Theory and Practice, v. 16 (Winter), p. 93-112, 1991.

LARSON, C. M.; CLUTE, R. C. The failure syndrome. American Journal of Small Business, v. 4, n. 2, p. 35-43, 1979.

LESSA, C. Micro, pequena e média empresa e a soberania nacional. Jornal Valor, p. A11, 22 dez. 2004.

LIEDHOLM, C.; MEAD, D. C. Small enterprises and economic development: the dynamics of micro and small enterprises. New York: Routledge, 1999.

LOVEMAN, G.; SENGENBERGER, W. In: SENGENBERGER, W.; LOVEMAN, G.; PIORE, M. J. (Eds.). The re-emergence of small enterprises: industrial re-structuring in industrialized countries. Geneve: Institut International d'Etudes Sociales, 1990.

MACHLUP, F. Theory of the firm: marginalist, behavioral, managerial. American Economic Review, v. 57, n. 1, p. 1-33, 1967.

MARCHESNAY, M. La mercatique de la petite entreprise. Revue Internationale PME, v. 1, p. 3-4, 1988.

MARCHINI, I. Piccola impresa e piccole imprese emergenti. Piccola Impresa, n. 1, p. 22-32, 1988.

MARSDEN, D. England (Capítulo). In: SENGENBERGER, W.; LOVEMAN, G.; PIORE, M. J. (Eds.). The re-emergence of small enterprises: industrial re-structuring in industrialized countries. Geneve: Institut International d'Etudes Sociales, 1990.

MARSHAL, A. Principles of Economics. London: MacMillan, [1920] 1961.

McMAHON, R. G. P.; HOLMES, S. Small business financial management practices in North America: a literature review. Journal of Small Business Management, v. 2, 1991.

MILLS, D. E.; SCHUMANN, L. Industry structure with fluctuating demand. American Economic Review, v. 75, n. 4, p. 758-767, 1985.

NAYAK, A.; GREENFIELD, S. The use of management accounting information for managing micro businesses. In: HUGUES, A.; STOREY, D. J. (Eds.). Finance and the small firm. London, 1994. 
PENROSE, E. The theory of the growth of the firm. Oxford: Basic Blackwell, 1959.

PIORE, M. J. The United States (Capítulo). In: SENGENBERGER, W.; LOVEMAN, G.; PIORE, M. J. (Eds.). The re-emergence of small enterprises: industrial re-structuring in industrialized countries. Geneve: Institut International d'Etudes Sociales, 1990.

; SABEL, C. The second industrial divide. New York: Basic Books, 1984.

POTIER, M. Quel Espace Economique Pour les PME. In: JULIEN, P. A.; CHICHA, J.; JOYAL, A. (Eds.). La PME dans un monde en mutation. Quebec: Presses de l'Université du Quebec, 1986.

POMBO, P.; HERRERO, A. Los sistemas de garantia para la micro y la pyme en una economia globalizada. Espanha: DP Editorial, 2001.

POTOBSKY, G. Small and medium-sized enterprises and labor law. International Labor Review, v. 13, n. 6, p. 601-628, 1992.

PRADO, A. Campanha de desburocratização e desregulamentação. Estudos Sebrae, ano 2, n. 11, p. 23-31, 1995.

PRESTON, L. The world of small business: a suggested typology. American Journal of Small Business, abr. 1977.

RATTNER, H. Introdução. In: RATTNER, HENRIQUE (Ed.). Pequena empresa. $O$ comportamento empresarial na acumulação e luta pela sobrevivência. São Paulo: Brasiliense, 1985.

ROBINSON, P. B.; HUNT, H. K. Autonomy as a motivational force in small businesses and entrepreneurship. In: JOHNSON, D. J. (Ed.). Small business - adapting for success in a changing world. Proceedings of The 1988 National Conference of The Small Business Institute Directors, v. 1, p. 195, 1988. Disponível em: <http://www.sbaer.uca.edu/Research/1988/88sbi192.txt>. Acesso em: 20 ago. 2005.

SCHERER, F. M. Industrial market structure and economic performance. Chicago: Rand McNally, 1980.

SCHUMPETER, J. Capitalism, Socialism, and Democracy. New York: Harper \& Row, 1942.

SCITOVSKY, I. Two concepts of external economies. In: AGARWALA, A.; SINGH, S. (Eds.). The economies of underdevelopment. New York: St. Martin, 1958.

SEBRAE - Serviço Brasileiro de Apoio às Micro e Pequenas Empresas. Desregulamentação e simplificação de procedimentos para as micro e pequenas empresas. Estudos e Pesquisas, ano 1, n. 1, 1994.

Pesquisa conjuntural. Estudos e Pesquisas, ano 2, n. 11, p. 14-21, 1995.

SFORZI, C. The quantitative importance of Marshallian industrial districts in the Italian economy. Paper given at the INTERNATIONAL CONFERENCE ON INDUSTRIAL DISTRICTS AND INTER-FIRM COOPERATION. 12-14 abr. Italy: University of Florence, 1989.

SMITH, A. The wealth of nations. New York: Random House, [1776] 1965.

SOLIMEO, M. D. As PMEs e a retomada do desenvolvimento. Estudos Sebrae, ano 1, n. 5 , p. 7-19, 1994. 
SOLOMON, G. T.; WEAVER, K. M.; FERNALD Jr., L. W. Historical examination of small business management and entrepreneurship pedagogy. Simulation \& Gaming, v. 25, n. 3, p. 338-352, set. 1994.

STEWART Jr., W. H.; ROTH, P. L. Risk propensity differences between entrepreneurs and managers: a meta-analytic review. Working Paper, n. 99-101. Arthur M. Spiro Center for Entrepreneurial Leadership, Clemson University, 1999. Disponível em: <http:/ /business.clemson.edu/Spiro/images/pdf/WP99-101.pdf>. Acesso em: 10 ago. 2005.

TAMARI, M. The financial structure of the small firm: an international comparison of corporate accounts in the U.S.A., France, U.K., Israel and Japan. American Journal of Small Business, v. 4, n. 4, p. 20-34, abr.-jun. 1980.

TOMMASO, M. R.; DUBBINI, S. Towards a theory of the small firm: theoretical aspects and some policy implications. United Nations, Economic Commission for Latin America and Caribbean, Division of Production, Productivity, and Development, 2000.

UNITED STATES SMALL BUSINESS ADMINISTRATION. The state of small business: a report of the President. Washington, DC, US Government Printing Office, 1997. VARGAS, G. Les crises de croissance de la PMI-PME. Revue Française de Gestion, jan.fev. 1984.

VEBLEN, T. The theory of the leisure class: an economic study of institutions. New York: New American Library, 1912.

VESPER, K. Strategic management and organization types: commentary. In: SCHENDEL, D. E.; HOFER, C. W. (Eds.). Strategic management. Englewood cliffs: Prentince Hall, 1979.

WAGNER, J.; STERNBERG, R. Personal and regional determinants of entrepreneurial activities: empirical evidence from the REM Germany. Working Paper, n. 624, University of Lueneburg, Bonn, 2002. Disponível em: <http://d.repec.org/ $\mathrm{n}$ ?u=RePec?iza:izadps:dp624>. Acesso em: 10 jul. 2005.

WEBSTER, F. A Model for new venture initiation. Academy of Management Review, v. 1, n. 1, 1976.

WEIMER, S. Germany (Capítulo). In: SENGENBERGER, W.; LOVEMAN, G.; PIORE, M. J. (Eds.). The re-emergence of small enterprises: industrial re-structuring in industrialized countries. Geneve: Institut International d'Etudes Sociales, 1990.

Recebido em 08 ago. 2005 Aprovação final em 11 out. 2005 